Gynecologic and

Obstetric Investigation
Gynecol Obstet Invest 2016;81:215-219

DOI: $10.1159 / 000440802$
Received: April 23, 2015

Accepted after revision: August 24, 2015

Published online: October 21, 2015

\title{
Patterns of First Recurrence in African American Patients with High Grade Epithelial Ovarian Carcinoma
}

\author{
Sareena Singh ${ }^{a, b} \quad$ Amy Armstrong $^{a, b} \quad$ Gaetan Pettigrew $^{b}$ Kimberly Resnick $^{a, b}$ \\ ${ }^{a}$ Division of Gynecologic Oncology and b Department of Obstetrics and Gynecology, University Hospitals Case \\ Medical Center, Cleveland, Ohio., USA
}

\section{Key Words}

African American · Ovarian cancer · Recurrence

\begin{abstract}
Background/Aims: The aim of this study is to compare the distribution of anatomic sites of first recurrence in African American (AA) patients with ovarian carcinoma compared to Caucasians. Methods: Patients diagnosed with high-grade epithelial ovarian, fallopian tube or peritoneal carcinoma from 2007 to 2013 were identified. Patterns of recurrence were compared for AA and Caucasian patients. Progressionfree survival (PFS) and overall survival (OS) were compared. Results: A total of 238 patients were included - 210 Caucasians and 28 AAs. At a follow-up time of 28 months, AAs were more likely to have multiple anatomic sites of recurrence rather than a single site when compared to Caucasians (63.6 vs. $35.5 \%, p=0.01$ ). Time to first recurrence was shorter for AA patients (12 vs. 18 months, $p<0.01$ ). PFS and OS did not differ. AA patients with multiple sites of first recurrence had a significantly shorter OS than Caucasian patients with multiple sites of first recurrence ( 24 vs. 30 months, $p=0.022$ ). Conclusion: Patterns of first recurrence differ between AAs and Caucasians. AAs have shorter times to first recurrence and are more likely to have multiple anatomic sites involved. AA patients with multiple sites of recurrence have a shorter OS than Caucasian patients with multiple sites.
\end{abstract}

(c) 2015 S. Karger AG, Basel

\section{KARGER}

(c) 2015 S. Karger AG, Basel

0378-7346/15/0813-0215\$39.50/0

E-Mail karger@karger.com

www.karger.com/goi

\section{Introduction}

In 2014, it was estimated that over 21,000 women will be diagnosed with ovarian cancer and over 14,000 women will die from the disease [1]. While not the most common type of gynecologic cancer diagnosed in the United States, ovarian cancer is the most fatal. The majority of patients are diagnosed with advanced stage disease, since symptoms are vague and no adequate screening strategies exist. It has been demonstrated that for many types of cancers African American (AA) patients have a lower stage-specific survival than Caucasians [2]. This also holds true for ovarian cancer. In a population-based SEER database study from 1988 to 1997, overall survival (OS) outcomes for 12,285 Caucasian women were compared to $798 \mathrm{AA}$ women with ovarian cancer [3]. AA women were found to have a median OS approximately 1 year shorter than Caucasian women. Data from the $\mathrm{Na}$ tional Center for Health Statistics and the National Cancer Institute (NCI) demonstrated that the 5-year survival rate for Caucasian women from 1974 to 2004 increased from 37 to $45 \%$, while the survival rate for AA women decreased from 43 to $38 \%$ [4]. Multiple studies have looked into why there exists such a discrepancy in survival and have concluded that a number of social, economic, cultural and health system issues are contributing factors [4-6]. 
Table 1. Patient and tumor characteristics

\begin{tabular}{lccc}
\hline & Caucasian $(\mathrm{n}=210)$ & AA $(\mathrm{n}=28)$ & p value \\
\hline Median age & $63(31-93)$ & $63.5(40-87)$ & 0.78 \\
Median BMI & $26.7(15.5-61.5)$ & $27.9(19.2-53)$ & 0.37 \\
Stage & & & 0.87 \\
$\quad$ I & $14(6.7)$ & $1(3.6)$ & \\
II & $5(2.4)$ & $1(3.6)$ & 0.39 \\
III & $174(82.9)$ & $23(82.1)$ & 0.60 \\
IV & $17(8.1)$ & $3(10.7)$ & \\
Median pre-operative CA-125 & $524.8(7.9-4,891)$ & $726.6(4.9-4,000)$ & \\
Debulking status* & $112(57.1)$ & $14(51.9)$ & 0.76 \\
Optimal & $84(42.9)$ & $13(48.1)$ & \\
Microscopic & & & \\
Histologic subtype & $172(81.9)$ & $25(89.3)$ & $0(0)$ \\
$\quad$ Serous & $4(1.9)$ & $2(8.7)$ & $1(3.6)$ \\
Mucinous & $21(10.0)$ & & \\
Clear cell & $13(6.2)$ & & \\
$\quad$ Endometrioid & & & \\
\hline
\end{tabular}

* For patients with $\geq$ stage II disease.

The vast majority of patients with ovarian cancer will recur, even if they have a complete response after surgical cytoreduction and platinum-based therapies. Since it has been established that there exists a survival differential in this patient population based on race and that the majority of patients recur despite initial responses to treatment, we sought to formally compare the patterns of recurrence in AA versus Caucasian patients with platinum-sensitive high grade epithelial carcinoma of the ovary, fallopian tube and peritoneum.

\section{Materials and Methods}

We conducted a retrospective case-control study of all patients diagnosed with high-grade epithelial ovarian, fallopian tube and peritoneal carcinoma. Patients were identified from an existing institutional database at the University Hospitals Case Medical Center. We included patients whose diagnosis was made between January 1, 2007, and December 31, 2013. Only patients who underwent complete surgical staging (hysterectomy, bilateral salpingo-oophorectomy, retroperitoneal lymph node dissection and omentectomy) and/or optimal surgical cytoreduction (defined as residual disease $\leq 1 \mathrm{~cm}$ in maximum diameter), who also received adjuvant platinum-based chemotherapy, were analyzed. Patients were excluded if medical records were incomplete or if anatomic location of first documented cancer recurrence was unable to be determined.

Patient demographics, tumor characteristics (histology, grade and stage), pre-operative CA-125 levels, surgical outcome and adjuvant chemotherapy details and responses were identified. First recurrence was determined by CA-125 levels, radiologic findings, pathology or operative report details. Anatomic locations of recurrence were identified as pelvic, abdominal, retroperitoneal lymph node, thoracic or other distant. Data regarding single versus multiple sites of first recurrence were also documented. Patients were considered to have multiple sites of recurrence if recurrence was detected in more than one anatomic location (pelvic, abdominal, retroperitoneal lymph node, thoracic or other distant). Progression-free survival (PFS) was determined from the time of surgery to the date of first recurrence. OS was calculated from the time of surgery to the date of last follow-up or date of death.

Patient characteristics between the 2 groups (AA and Caucasian) were compared using the Fisher exact test and the $\chi^{2}$ test for categorical variables and the $t$ test and the Mann-Whitney $U$ test for continuous variables. The Kaplan-Meier method was used to calculate OS and PFS. A 2-sided p value of $<0.05$ was considered statistically significant. SPSS version 22 (IBM, Chicago, Ill., USA) was used for statistical analyses.

\section{Results}

A total of 238 patients who underwent surgical cytoreduction or complete surgical staging for high-grade epithelial ovarian cancer were identified. Of these patients, 210 were Caucasian and 28 were AA. All patients had a GOG performance status of $0-1$, and $91.2 \%$ of patients completed 6 cycles of adjuvant platinum-based chemotherapy. Patient and tumor characteristics are listed in table 1. Groups did not differ significantly with regards to age, body mass index and pre-operative CA-125 level. Most patients (82.7\%) had stage III disease. The most 
Table 2. Anatomic patterns of first recurrence

\begin{tabular}{llrl}
\hline & $\begin{array}{l}\text { Caucasian } \\
(\mathrm{n}=154)\end{array}$ & $\begin{array}{l}\text { AA } \\
(\mathrm{n}=22)\end{array}$ & $\begin{array}{l}\mathrm{p} \\
\text { value }\end{array}$ \\
\hline $\begin{array}{l}\text { Anatomic location of recurrence } \\
\quad \text { Pelvis }\end{array}$ & $78(44.3)$ & $10(45.5)$ & 0.91 \\
$\quad$ Abdomen & $48(27.3)$ & $8(36.4)$ & 0.37 \\
Retroperitoneal lymph node & $69(39.2)$ & $12(54.5)$ & 0.17 \\
$\quad$ Thoracic & $26(14.8)$ & $7(31.8)$ & 0.04 \\
$\quad$ Other distant & $27(15.3)$ & $4(18.2)$ & 0.76 \\
Number of recurrence sites & & & 0.01 \\
$\quad$ Single & $92(64.8)$ & $8(36.4)$ & \\
$\quad$ Multiple & $62(35.2)$ & $14(63.6)$ & \\
\hline
\end{tabular}

Data are presented as n (\%).

common histologic subtype was high-grade serous carcinoma, with $82.7 \%$ of included patients having this subtype. Stage and histologic subtype did not differ between the 2 groups.

The median follow-up time was 28 months (7-67 months). At this median follow-up time, 176 (74\%) of patients had experienced a cancer recurrence. Of the patients with a recurrence, 22 were AA and 154 were Caucasian. Information regarding anatomic sites of first recurrence is summarized in table 2 . For the $22 \mathrm{AA}$ patients with a recurrence, $14(63.6 \%)$ had multiple anatomic sites of recurrence. This differed significantly when compared to Caucasian patients, of which only $62(35.2 \%)$ had multiple anatomic sites involved at the time of first recurrence $(\mathrm{p}=0.01)$. AA patients were also more likely to have thoracic involvement than Caucasian patients (31.8 vs. $14.8 \%, \mathrm{p}=0.04)$. Groups did not differ significantly with regards to other anatomic recurrence sites (pelvis, abdomen and retroperitoneal lymph nodes). Median time to diagnosis of first recurrence was significantly shorter for the AA group (12 months [6-16]) than for the Caucasian group (18 months [6-32]; $\mathrm{p}<0.01$ ).

After a median follow-up time of 28 months for all patients, the median PFS for AA patients (12 months, 95\% CI 10.7-13.3) did not differ significantly when compared to Caucasian patients (19 months, 95\% CI 18.2-19.8; $\mathrm{p}=$ 0.171 ; fig. 1). The median OS for AA patients (30 months, 95\% CI 26.9-33.0) also did not differ significantly when compared to Caucasian patients (38 months, 95\% CI 35.9-40.1; $\mathrm{p}=0.078$; fig. 2). OS was compared by race for patients who had multiple anatomic sites involved when diagnosed with first cancer recurrence. For this comparison, median OS for AA patients with multiple sites of first recurrence (24 months, 95\% CI 21.6-26.4) was signifi-

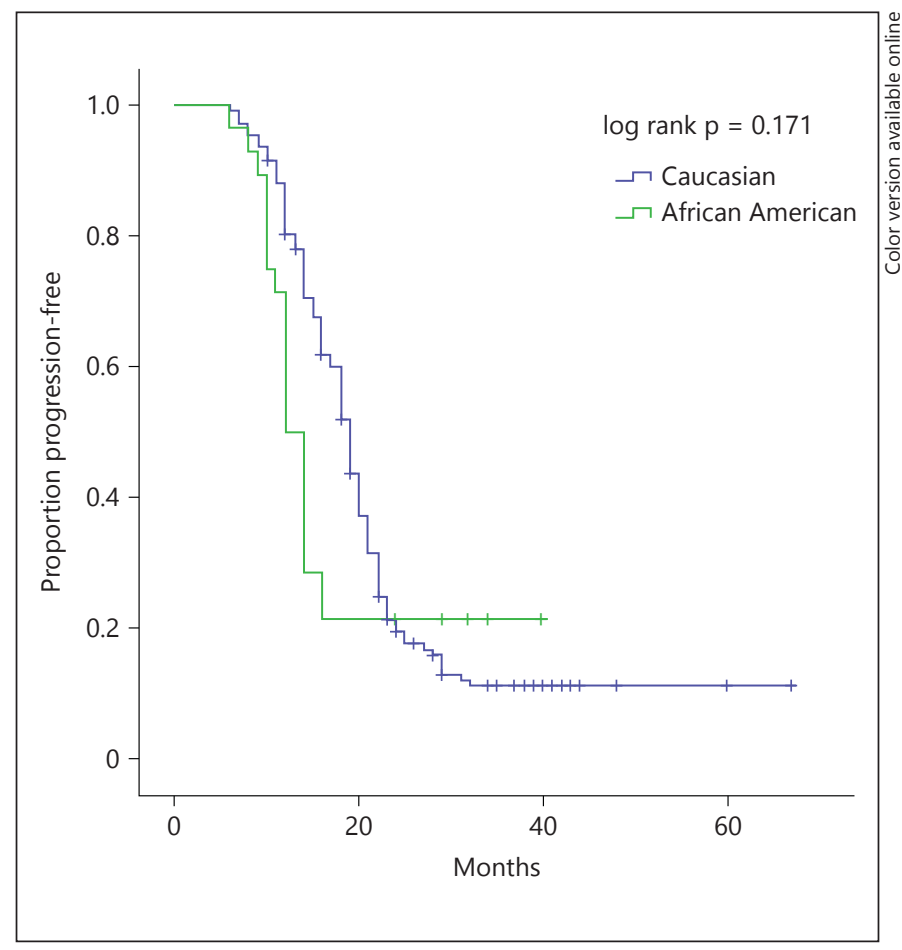

Fig. 1. Kaplan-Meier curves for PFS by race.

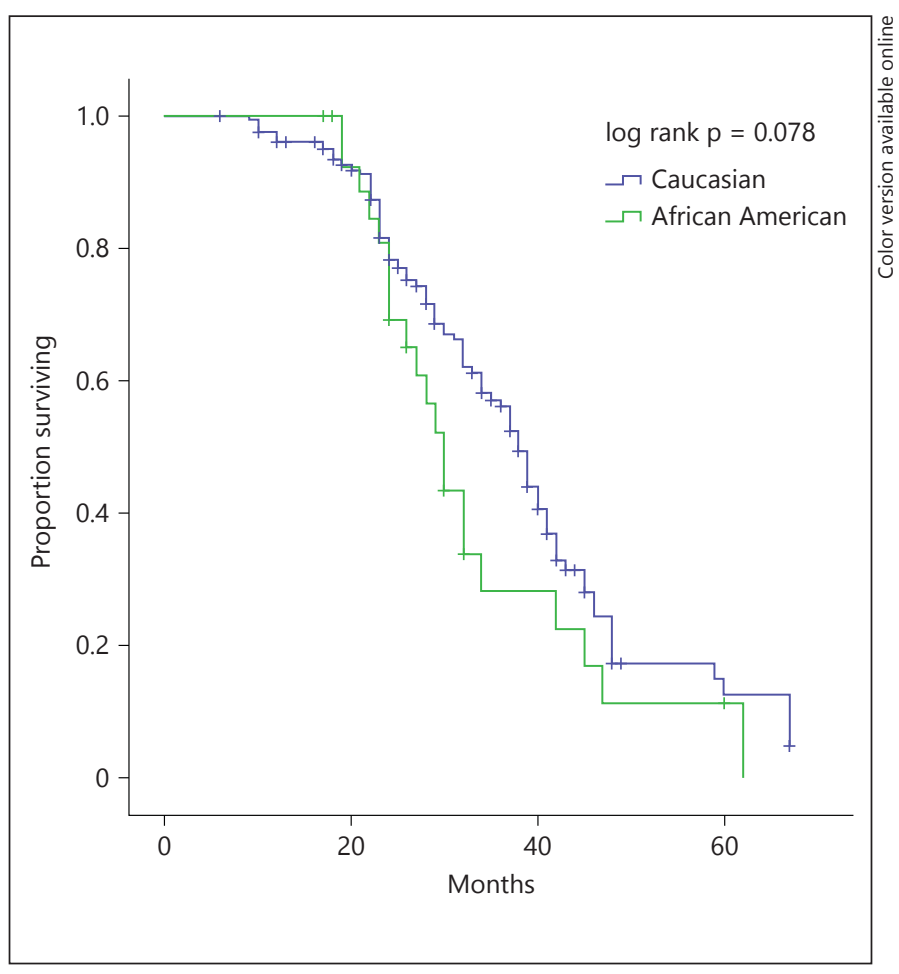

Fig. 2. Kaplan-Meier curves for OS by race. 


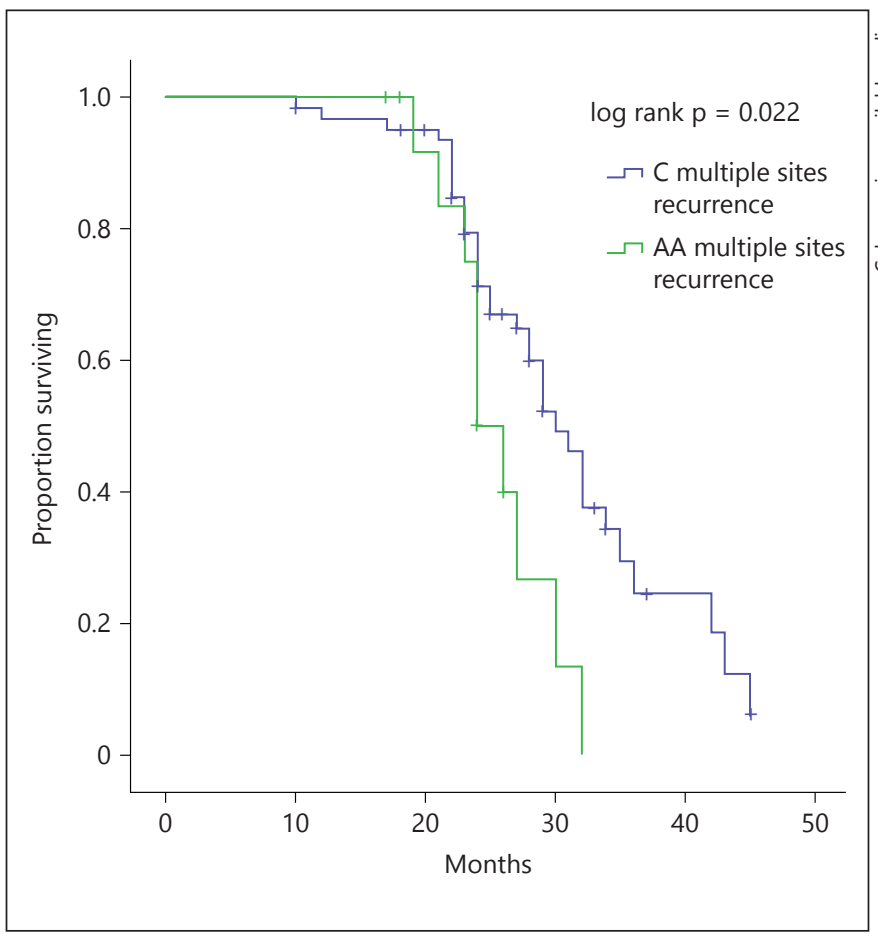

Fig. 3. Kaplan-Meier curves for OS by race for patients with multiple anatomic sites involved at time of diagnosis of first recurrence.

cantly shorter than Caucasian patients with multiple sites of first recurrence (30 months, 95\% CI 27.3-32.6; $\mathrm{p}=$ 0.022; fig. 3).

\section{Discussion}

Disparities in survival of AA patients with ovarian cancer as compared to Caucasian patients have been established through a number of single-institution and national database reviews. Such discrepancies have been attributed to a number of factors, including tumor biology and socioeconomic influences [4-6]. This is the first study to specifically examine the patterns of cancer recurrence in AA patients and to further investigate how cancer recurrence patterns affect oncologic outcomes. In this study, we have shown that AA patients are more likely to have the involvement of multiple anatomic sites when diagnosed with a first recurrence and that AA patients are also more likely to have disease in the chest at time of first recurrence. The PFS and OS for patients in our study did not differ significantly when stratified by race. However, AA patients with multiple anatomic sites of first recurrence have shorter OS times than Caucasian patients. We also have demonstrated that time to detection of first recurrence is shorter for AA patients than it is for Caucasian patients.

Bristow et al. [7] published an analysis of racial disparities in stage III ovarian cancer on oncologic outcomes. In this retrospective review, the authors examined the effects of treatment regimens that included surgery and chemotherapy on OS according to race. A total of 405 patients were included in this review. On multivariate analysis, increasing age, high-grade tumors, administration of platinum-based chemotherapy and no gross residual disease after surgical cytoreduction were all found to be independently associated with OS. Race, however, was found not to be associated independently with OS (hazards ratio 1.06, 95\% CI 0.61-1.79). The authors concluded that when equal access to care was provided, the survival disparities shown in previous population-based studies were eliminated. Our study included patients with similar age, stage and histology distributions who were treated at a NCI-designated cancer center. While we did not demonstrate a difference in OS or PFS by race classification alone, we did, however, demonstrate differences in specific recurrence.

The scope of this problem is larger than can be addressed in this discussion. Previously, a variety of socioeconomic status (SES), psychosocial and biologic reasons for survival differences by race classification have been offered to explain disparities in oncologic outcomes [35]. The vast majority of research has been performed in breast and prostate malignancies. Extrapolation from these disease sites is necessary. In no other disease site is the disparity quite as evident as in prostate cancer. A SEER study of patients with prostate cancer demonstrated longer time from diagnosis to commencement of all treatment types when comparing AA versus white patients [8]. Even after the start of treatment, AAs demonstrate higher rates of recurrence, even among low-risk prostate cancers, suggesting a biologic rationale for such differences [9]. Palmer et al. [10] recently described barriers to care among 203 breast cancer survivors. Compared to white survivors, AA survivors were more likely to identify cost, out-of-pocket expenses and anxiety regarding transportation as barriers to care. Looking beyond psychosocial and SES factors, Lindner et al. [11] describe molecular differences in transcription factors and tumor angiogenesis in triple negative subtypes of breast cancer between AA and white patients. An interesting and futuristic conclusion of this study would be 'personalized cancer care' based on race at the genotypic level. 
In our study, we also found that having multiple anatomic sites of recurrence was associated with a worse OS compared to patients with single sites of recurrence. This is consistent with previous studies, which have examined outcomes for patients with recurrent ovarian cancer [12, 13]. Both of these studies looked at the benefit of secondary surgical cytoreduction for patients with recurrent disease. Chi et al. [12] found that patients with a single site of recurrence had a greater OS than patients with multiple sites of recurrence and patients with carcinomatosis (60 vs. 42 vs. 28 months, respectively, $\mathrm{p}<0.001)$. Similarly, Salani et al. [13] showed that patients with 1-2 sites of recurrence had a greater median OS than patients with $3-5$ sites of cancer recurrence ( 50 vs. 12 months, $p<0.03$ ). These 2 studies, however, did not specifically report oncologic outcomes of patients with recurrent disease based on race. These studies also did not specifically include details regarding how cancer recurrence was treated (chemotherapy and/or surgery). This provides us with an additional question for further investigations.

Our study is limited by the retrospective nature of its design and the biases that are inherent to such studies. Only $12 \%$ of patients included in the study were AA. This is a small percentage; however, this can be attributed to the relative rarity of ovarian cancer in the AA population as a whole. Data were also collected from a single institution and, therefore, may not account for potential differences in practice patterns. Strengths of this review include this being the only study to specifically analyze recurrence patterns of patients with ovarian cancer as stratified by race. Also, all patients included in our study were treated by gynecologic oncology sub-specialists at an NCIdesignated cancer institute.

Disparities in healthcare outcomes among different ethnic and socioeconomic groups are a sizeable challenge with no panacea. Further elucidation of differences at the genotypic level is needed in order to target therapy and further champion 'personalizing medicine'. Physician and staff cultural competency is needed to make certain that the psychosocial and SES stressors faced by our patients do not hinder their ability to achieve outcomes equal to their Caucasian peers. Prospective trials are needed to validate our initial findings and truly define the effects of recurrence patterns on survival in patients with ovarian cancer.

\section{Disclosure Statement}

The authors have no financial conflicts of interest to disclose.

\section{References}

1 American Cancer Society: Cancer Facts \& Figures 2014. Atlanta, American Cancer Society, 2014.

2 Bach PB, Schrag D, Brawley OW, et al: Survival of blacks and whites after a cancer diagnosis. JAMA 2002;287:2106-2113.

-3 Barnholtz-Sloan JS, Tainsky MA, Abrams J, et al: Ethnic differences in survival among women with ovarian carcinoma. Cancer 2002;94: 1886-1893.

4 Institute of Medicine: The Unequal Burden of Cancer. Washington, National Academy Press, 1999.

5 Institute of Medicine: Unequal Treatment: Confronting Racial and Ethnic Disparities in Healthcare. Washington, National Academy of Press, 2003.

6 Freeman HP: Poverty, culture, and social injustice: determinants of cancer disparities. CA Cancer J Clin 2004;54:72-77.
Bristow RE, Ueda S, Gerardi MA, et al: Analysis of racial disparities in stage IIIC epithelial ovarian cancer care and outcomes in a tertiary gynecologic oncology referral center. Gynecol Oncol 2011;122:319-323.

8 Stokes WA, Hendrix LH, Royce TJ, et al: Racial differences in time from prostate cancer diagnosis to treatment initiation: a population-based study. Cancer 2013;119:24862493.

-9 Sundi D, Ross AE, Humphreys EB, et al: African American men with very low-risk prostate cancer exhibit adverse oncologic outcomes after radical prostatectomy: should active surveillance still be an option for them? J Clin Oncol 2013;31:2991-2997.
10 Palmer NR, Weaver KE, Hauser SP, et al: Disparities in barriers to follow-up care between African American and white breast cancer survivors. Support Care Cancer 2015;23: 3201-3209.

11 Lindner R, Sullivan C, Offor O, et al: Molecular phenotypes in triple negative breast cancer from African American patients suggest targets for therapy. PLoS One 2013;8:e71915.

12 Chi DS, McCaughty K, Diaz JP, et al: Guidelines and selection criteria for secondary cytoreductive surgery in patients with recurrent, platinum-sensitive epithelial ovarian carcinoma. Cancer 2006;106:1933-1939.

13 Salani R, Santillan A, Zahurak ML, et al: Secondary cytoreductive surgery for localized, recurrent epithelial ovarian cancer: analysis of prognostic factors and survival outcome. Cancer 2007;109:685-691. 